www.nature.com/ijo

\title{
EDITORIAL
}

\section{Promise and limitations of functional neuroimaging in the study of obesity: is it time for a consortium and a multicenter trial?}

\author{
International Journal of Obesity (2009) 33, 607-610; \\ doi:10.1038/ijo.2009.55
}

In the last 10 years functional neuroimaging (FN), including positron emission tomography (PET), functional magnetic resonance imaging (fMRI) and magnetoencephalography (MEG), has been generating, at an increasing pace, data on human eating behavior and its aberrations leading to weight gain and obesity. Today, several obesity centers consider neuroimaging as one of the most promising tools for studying the pathophysiology of obesity in humans. This success is mainly the result of two factors: (1) the experimental notion that the central nervous system (CNS) is the master regulator of energy balance and eating behavior, as supported by decades of research in animals ${ }^{1}$ and sporadic cases of CNS lesions in humans associated with obesity, ${ }^{2}$ and (2) the limited direct access to the CNS for scientific experiments in humans that imposes the use of non-invasive methodologies, such as functional neuroimaging, for the study of the in vivo physiology of the organ.

On the other hand, the application of FN techniques for the investigation of human obesity encounters limitations partly related to the understanding of the pathophysiology of weight gain and obesity, and partly related to the methodological constraints of the FN technology. ${ }^{3}$ In fact, although it is clear that the thermodynamic basis for weight gain is a protracted positive energy balance, the identification of the etiopathogenetic factors determining weight gain is still controversial and reflects the multiplicity of metabolic conditions and behavioral phenotypes associated with the development of obesity. ${ }^{4}$

From a methodological standpoint, FN is a versatile technology that can be used to map the distribution of specific molecules bound to radioligands (PET) or to map the regions of the brain that respond to a specific stimulation either by measuring surrogate markers of regional blood flow (blood oxygen level dependent (BOLD)) or arterial spin labeling (ASL)-fMRI or ${ }^{15} \mathrm{O}$-PET, or by measuring neural

'Neuroimaging in Obesity Research' sponsored by the Obesity Taskforce of the National Institutes of Health in Bethesda (MD, USA) on 27-28 October 2008 electric activity (MEG). Trade-offs between a priori molecular hypotheses and exploratory versatility, with consequences on the sensitivity and specificity of the measurements, inform the choice of an FN technique vs another in a determined study. For example, BOLD-fMRI can be used to scan the entire brain at once, exploring the brain responses to a specific stimulation on a regional level with no a priori restrictions to regions or molecular networks of interest. However, BOLD-fMRI cannot identify the specific molecular mediators of those brain responses, which could potentially be identified by a radioligand-PET study.

From a management standpoint, FN studies are resource intensive and logistically complex. First, they require the use of costly imaging facilities and expert staff, often overloaded with clinical work and not always conveniently located for the obesity centers. Second, imaging studies of obese subjects are limited by the weight tolerance of the scanner tables and by the diameter of the transversal section of the bore of the MRI scanners. In a recent study, two PET technologists reported that in a large sample of clinical studies with obese subjects, problems ranged from difficult intravenous access to patient positioning and motion, which ultimately required extra time to be spent near the patient. ${ }^{5}$ Cost, logistics and operational demands can explain why the sample size of most published FN studies does not include more than 10 obese subjects.

Despite all these limitations, the neuroimaging investigation of obesity has progressed from the initial mapping exercises to hypotheses-testing studies, suggesting multiple neurofunctional markers of eating behavior and obesity. In fact, by investigating the whole brain at once, FN studies have identified these markers in a wide array of brain regions, spanning the cortical and subcortical levels. Besides the candidate areas supported by overwhelming evidence in laboratory animals (hypothalamus and brainstem), FN has drawn attention to the role of the prefrontal and orbitofrontal cortices, striatum, insula and other paralimbic regions as brain areas implicated in processing responses to food consumption or food perception, and to the relative deficiency of dopamine type 2 receptors as underlying human obesity. ${ }^{6}$ This has supported the appreciation of the neurobiological bases of obesity and, at the same time, has documented the complexity of the neural substrates under- 
lying the interplay among cognitive, hedonic and metabolic processes regulating eating behavior in humans. Therefore, FN has corroborated the behavioral evidence that human eating is, in fact, an idiosyncratic behavior, driven not only by the need to compensate for acute and chronic energy imbalances, but also by emotional, cultural and cognitive factors often overriding energy homeostasis in the initiation and termination of a meal or a snack.

In terms of experimental settings, when we look at the evidence accrued so far, we notice that most BOLDfMRI studies have actually used the exposure to food sensory cues, most commonly visual cues, as the stimulus triggering the imaged brain response. There is no doubt that sight, as well as olfaction, taste and the mouth feel of food are the fundamental means for the sensorial appreciation of food and as such function as the screeners, gatekeepers and primary reinforcers of eating choices. At the same time, the sensory experience of a forthcoming meal is conducive to physiological and psychological phenomena (cephalic phase response, expectation of reward), which prepare the organism for the ingestion of food. In addition, for a number of technical and logistic reasons, sensorial stimulation is experimentally suitable for current FN settings. The combination of physiological rationale and experimental convenience has established sensorial stimulation as the paradigm of choice in BOLDfMRI studies of eating behavior and obesity.

However, although the study of the brain responses to food sensory cues has yielded a fertile output, it is the consumption of a meal constituting the event that, more than anything else, marks the pace of human eating behavior and represents the outcome of a combination of motivational (and as such also sensorial), cognitive and metabolic factors. The frequency and caloric content of meals are the two single quantitative factors that determine the amount of energy intake. In particular, the caloric content of a meal is the natural target of dietetic interventions for weight loss, whereas manipulations of the meal volume and its macronutrient composition with less energydense foods are often used instrumentally to elicit satiety in response to a reduced energy intake. Despite this compelling evidence for meal consumption as the main eating behavior phenomenon of interest, the investigation of the brain responses to the ingestion of a meal has been pursued much less than the investigation of the brain responses to food sensory cues. This is mainly because the consumption of food presents several technical challenges in a functional neuroimaging setting, from a design and logistic point of view.

A complementary approach is to combine 'sensorial' FN studies with eating behavioral variables, including measures of food intake, behavioral phenotypes and visual analog scale assessments of subjective states, such as hunger, fullness, desire to eat and so on. Although several examples of this approach have already generated interesting results, ${ }^{7,8}$ more remains to be explored and understood about the concordance between objective (for example, FN changes in regional brain activity) and subjective variables of eating behavior and metabolic states.

Nevertheless, in the course of the last 10 years, the field has evolved from the stage of a pioneering exploration of the neuroanatomical correlates of eating behavior and obesity, ${ }^{3,6}$ which described genuinely novel findings, to a second generation of studies, which tested specific regions of interest hypotheses and investigated their relevance to the pathophysiology of weight gain and obesity. In fact, a study in formerly obese individuals (so-called 'post-obese', who had normalized their body weight from an earlier obese status only through diet and physical exercise) demonstrated that some of the neurofunctional differences between obese and normal-weight individuals persisted in post-obese individuals to indicate the possible presence of neurofunctional markers of increased risk for obesity, ${ }^{9}$ a finding that warrants testing in longitudinal studies. Another study in successful dieters (that is, dieters who had lost a large amount of body mass and kept their normal weight status) confirmed the role of the dorsal prefrontal cortex as the brain region orchestrating the cognitive control over eating behavior and as such neurofunctionally correlated with dietary restraint, ${ }^{7}$ a finding also supported by FN studies in people affected by anorexia nervosa. ${ }^{10}$

At the same time, administration of orexigenic (that is, ghrelin) $^{11}$ and anorexigenic hormones (that is, leptin) ${ }^{12}$ has been used as an experimental paradigm to test how in different metabolic conditions the brain response to visual food cues is modulated by the exogenous manipulation of endocrine signals mimicking changes in energy balance. Two studies ${ }^{13,14}$ have also described the effects of leptin administration on the brain response to visual food cues in leptin-deficient individuals, who are affected by a very rare genetic form of extreme obesity, though highly responsive to leptin treatment. Another study ${ }^{8}$ described the brain response to the administration of $\mathrm{PYY}_{3-36}$, an anorexigenic hormone, in normal-weight individuals, also showing that some changes in local neural activity were correlated with subsequent food intake. All these pharmacological (by the administration of so-called 'biologics') intervention studies have confirmed that the central control of eating behavior is operated by brain regions spanning the cortical and subcortical levels, although the consistency of region-specific findings across studies is rather limited. ${ }^{8,11-14}$

All in all, many of the FN reports await confirmation in independent studies. On the other hand, differences in subject characteristics, study designs, scanners, data acquisition and analysis procedures across studies limit the utility of retrospective cross-validation of FN findings. Each of these sources of heterogeneity across studies is worth a specific dissertation and, evidently, reflects the combined effect of the limitations inherent in the current understanding of the pathophysiology of obesity and in the current capability and methodologies of FN. For illustration purposes, I would just 
mention three examples (among the many) for the differences in subject characteristics and comment on some sources of variability in data analysis.

For subject characteristics:

(1) The choice of variable degrees of obesity (classified by body mass index) for the disease group and of leanness for the control group is inconsistent across studies. The use of the BMI alone is also questionable as the classifier of obesity, particularly in small-sample studies, given the spread of body fatness that can be represented by the same BMI.

(2) The recent weight history of the subjects and, most importantly, the notion of their energy-balance status at the time of the study are often neglected in FN studies. In other words, it is often unknown whether the subjects are gaining, losing or keeping their body weight stable (and for how long) when they are studied.

(3) The presence of concomitant pharmacotherapy and/or physiological conditions that can alter eating behavior, for example the phase of the menstrual cycle in fertile women at the time of the study.

Regarding the data analysis, we know that locating a response in a determined region of the brain is a function of the anatomical space and of the regional and subregional demarcations that we adopt. As a result, the findings reported in the same cortical brain region may actually be quite distant in anatomical location; they may belong to diverse functional subregions, and therefore, possibly express different neurobiological phenomena. A first step towards improvement in this respect would be the execution of intra-subject reproducibility studies aimed at assessing the typicalness of a pattern of responses to a determined stimulus, and helping the assessment of the impact of random variables that can confound the results. A second step could be the execution of inter-subject reproducibility studies to assess the consistency of a certain brain response in a phenotypically homogenous group of subjects (for example, with the same body fatness, same age and same gender). In my personal experience, the attempt to reproduce responses to food consumption with ${ }^{15} \mathrm{O}$-PET in anthropometrically similar groups of subjects studied with the same experimental protocol failed, although confounding factors external to the subject groups studied could not be ruled out (Del Parigi A et al., unpublished observation).

On the other hand, the spatial resolution of current FN techniques and equipment is the main limiting factor for our ability to investigate neurofunctional responses in subdivisions of pivotal regions for the regulation of food intake, such as the hypothalamus and the brainstem. Although local changes of activity in these brain regions have been reported in several studies, the richness in specialized neuronal populations clustered in the nuclei with diverse and, in some cases, opposite functional output for the regulation of energy balance - as we have learned from rodent models of obesity - sets the bar for understanding the physiological meaning of local changes of activity at a scale (the individual nuclei) that scale - the indi is, arguably, still out of reach of the FN technology. The combination of mapping studies of local changes in neural activity with radioligandbinding studies can offer an access to the receptor molecular level, contingent upon the availability of specific radioligands for the receptors/pathways of interest. Likewise, the combination of mapping studies with molecular biology approaches, such as the study of transcriptomics in postmortem samples of cerebral tissue, recently proposed as 'neuroimagenomics', 15 could prove useful in bridging the gap between FN-detected changes in local neural activity and the underlying molecular biology. In this framework, studies of obesity in animal models can also be used as a flexible tool to develop novel radioligands and imaging techniques, to test mechanistic hypotheses, and to inform on the molecular machinery of the imaged effects and epiphenomena.

Inter-individual differences in brain regional morphology, and macro- and micro-organization add another layer of complexity to FN studies. Furthermore, the stereotactical location of a signal is affected by the data-processing algorithm, including the use of standard or customized anatomical templates, which can substantially affect the results of the data analysis. It is by personal experience that even minimal permutations in the data-analysis procedures, such as implementing additional steps in pre-processing the data, using different releases of the same data-analysis software, changing the value of one or more data-analysis parameters, still within acceptable ranges, or subtracting one subject (possible outlier) can change the results at the group level (Del Parigi et al., personal communication) and generate potentially conflicting publications. ${ }^{16,17}$

Taken together, the evidence accumulated by using FN in obesity research is at the same time relevant, promising and yet weak. It is relevant because it documents the feasibility of FN studies in obese people and the ability to identify putative neurofunctional markers of this condition. It is promising because it is a formidable tool for the exploration of the in vivo brain physiology and as such stimulates the interest of many obesity researchers. It is weak, however, because it is based on a wide array of findings, often bound to specific study designs and to specific study subject characteristics. Ultimately, for the most part, these findings await confirmation in independent studies in order to gain a certain degree of generalizability to the obese population.

\section{Next step}

In the face of these limitations and challenges, the step required to advance the field critically could be the implementation of a multicenter study, using the same protocol in different centers, each of them contributing to an overall sufficiently large sample size, meeting specific inclusion/ exclusion criteria, to warrant more generalizable results. 
To accomplish this, I propose the creation of an Obesity Neuroimaging Consortium, that is, a consortium among scientific institutions technically and scientifically equipped to use FN for the study of the pathophysiology of human obesity. A steering committee would have the task of designing a protocol, defining the characteristics of the study population and subgroups, setting the inclusion/ exclusion criteria, deciding the rules for data management and statistical analysis implemented in a central core institution, as well as for the writing of reports.

The protocol has to be conceived to address basic knowledge gaps (for example, the temporal course of the brain response to meal consumption, and the modulation of brain responses by different phenotypes and genotypes) and test the fundamental hypotheses of the central control of eating behavior (for example, the functional interrelations between the brainstem, the hypothalamus and the prefrontal cortex in controlling food intake during a meal), and its dysregulation leading to weight gain and obesity. At the same time, the protocol has to be simple and feasible, in order to be implemented in as many centers as are qualified to run FN studies and not unduly penalizing their capacity to recruit subjects.

In terms of statistical power and sample-size calculation, the additional variance contributed by a multicenter study compared with a single-center study is likely to be minimal, as assessed by a recent study, which, using theoretical models of power and empirical data, concluded that the between-center variability can be controlled and does not require a large compensatory addition of total subjects. ${ }^{18}$ Across-center differences in technical equipment, including scanners, field strength and performance, need to be taken into account as potential trade-offs when assessing the feasibility of the protocol.

From a data-analysis standpoint, as in other similar experiences, the centralization of data management helps in avoiding marginal discrepancies across sites in the implementation of data-analysis algorithms, and guarantees the achievement of a pre-defined and optimal quality standard.

Although highly resource demanding, an Obesity Neuroimaging Consortium has the potential to attract funding from multiple sources, including federal agencies and private donors and sponsors, such as biotechnology and pharmaceutical industries. A multicenter study with a sufficiently large sample size to answer basic questions on the central control of eating behavior in humans is, in fact, one of the best investments to advance the understanding of the pathophysiology of human obesity and guide the search for novel biomarkers of efficacy and potentially for novel molecular targets for the development of an effective pharmacotherapy of obesity, which is acutely needed.

A Del Parigi Academic Medicine, Pfizer Inc., New York, NY, USA E-mail: angelo.delparigi@pfizer.com

\section{References}

1 Morton GJ, Cummings DE, Baskin DG, Barsh GS, Schwartz MW. Central nervous system control of food intake and body weight. Nature 2006; 443: 289-295.

2 Bray GA. Obesity is a chronic, relapsing neurochemical disease. Int J Obes Relat Metab Disord 2004; 28: 34-38.

3 DelParigi A, Pannacciulli N, Le DN, Tataranni PA. In pursuit of neural risk factors for weight gain in humans. Neurobiol Aging 2005; 26 (Suppl 1): 50-55.

4 Keith SW, Redden DT, Katzmarzyk PT, Boggiano MM, Hanlon EC, Benca RM et al. Putative contributors to the secular increase in obesity: exploring the roads less traveled. Int J Obes (Lond) 2006; 30: 1585-1594.

5 Botkin CD, Osman MM. Prevalence, challenges, and solutions for (18)F-FDG PET studies of obese patients: a technologist's perspective. J Nucl Med Technol 2007; 35: 80-83.

6 Tataranni PA, DelParigi A. Functional neuroimaging: a new generation of human brain studies in obesity research. Obes Rev 2003; 4: 229-238.

7 DelParigi A, Chen K, Salbe AD, Hill JO, Wing RR, Reiman EM et al. Successful dieters have increased neural activity in cortical areas involved in the control of behavior. Int J Obes (Lond) 2007; 31: 440-448.

8 Batterham RL, Ffytche DH, Rosenthal JM, Zelaya FO, Barker GJ, Withers DJ et al. PYY modulation of cortical and hypothalamic brain areas predicts feeding behaviour in humans. Nature 2007; 450: 106-109.

9 DelParigi A, Chen K, Salbe AD, Hill JO, Wing RR, Reiman EM et al. Persistence of abnormal neural responses to a meal in postobese individuals. Int J Obes Relat Metab Disord 2004; 28: 370-377.

10 Uher R, Murphy T, Brammer MJ, Dalgleish T, Phillips ML, Ng VW et al. Medial prefrontal cortex activity associated with symptom provocation in eating disorders. Am J Psychiatry 2004; 161: 1238-1246.

11 Malik S, McGlone F, Bedrossian D, Dagher A. Ghrelin modulates brain activity in areas that control appetitive behavior. Cell Metab 2008; 7: 400-409.

12 Rosenbaum M, Sy M, Pavlovich K, Leibel RL, Hirsch J. Leptin reverses weight loss-induced changes in regional neural activity responses to visual food stimuli. J Clin Invest 2008; 118: 2583-2591.

13 Baicy K, London ED, Monterosso J, Wong ML, Delibasi T, Sharma A et al. Leptin replacement alters brain response to food cues in genetically leptin-deficient adults. Proc Natl Acad Sci USA 2007; 104: 18276-18279.

14 Farooqi IS, Bullmore E, Keogh J, Gillard J, O'Rahilly S, Fletcher PC. Leptin regulates striatal regions and human eating behavior. Science 2007; 317: 1355.

15 DelParigi A, Page GP, Allison DB, Beach TG, Stephan DA, Reiman EM et al. Neuroimagenomics for the Study of Human Obesity. Immun Endocr Metab Agents Med Chem 2006; 6: 75-80.

16 DelParigi A, Gautier JF, Chen K, Salbe AD, Ravussin E, Reiman E et al. Neuroimaging and obesity: mapping the brain responses to hunger and satiation in humans using positron emission tomography. Ann N Y Acad Sci 2002; 967: 389-397.

17 Le DS, Pannacciulli N, Chen K, DelParigi A, Salbe AD, Reiman EM et al. Less activation of the left dorsolateral prefrontal cortex in response to a meal: a feature of obesity. Am J Clin Nutr 2006; 84: 725-731.

18 Suckling J, Ohlssen D, Andrew C, Johnson G, Williams SC, Graves $\mathrm{M}$ et al. Components of variance in a multicentre functional MRI study and implications for calculation of statistical power. Hum Brain Mapp 2008; 29: 1111-1122. 